Textures and Microstructures, 1991, Vols 14-18, pp. 279-282 Reprints available from the publisher

Photocopying permitted by license only
C) 1991 Gordon and Breach Science Publishers SA Printed in the United Kingdom

\title{
NEUTRON TEXTURE INVESTIGATION OF A METEOR
}

K. HELMING ${ }^{*}$, A. N. NIKITIN ${ }^{\dagger}$, and K. WALTHER*

* Joint Institute for Nuclear Research, Laboratory for Neu-

+ tron Physics, 141980 Dubna, USSR

State Teachers' Training College, Lenin Prospect, 300026 Tula, USSR

INTRODUCTION

At the $17^{\text {th }}$ May 1990 near the Bashkirian town of Sterlitamak fell an iron-nickel meteor. For the first time the texture of a meteor has been measured. The knowledge of the texture of meteors may give absolutely new information upon deformation processes in cosmic bodies and would be useful for the cosmogony.

\section{EXPERIMENT}

The material of the meteor was investigated by neutron diffraction at the steady state reactor of the Central Institute for Nuclear Research Rossendorf. A sample with nearly cubic shape was cut with a spark erosive saw from a meteor fragment. The material has many internal fissures.

The analysis of the neutronographic pattern shows a bcc structure with a lattice constant $a_{0}=2.86 \AA$. The neutron wavelength of the monochromated beam is $\lambda=1.437 \AA$. The 
transmission part of the (110), (200), and (211) pole figures has been measured (see fig. 1).
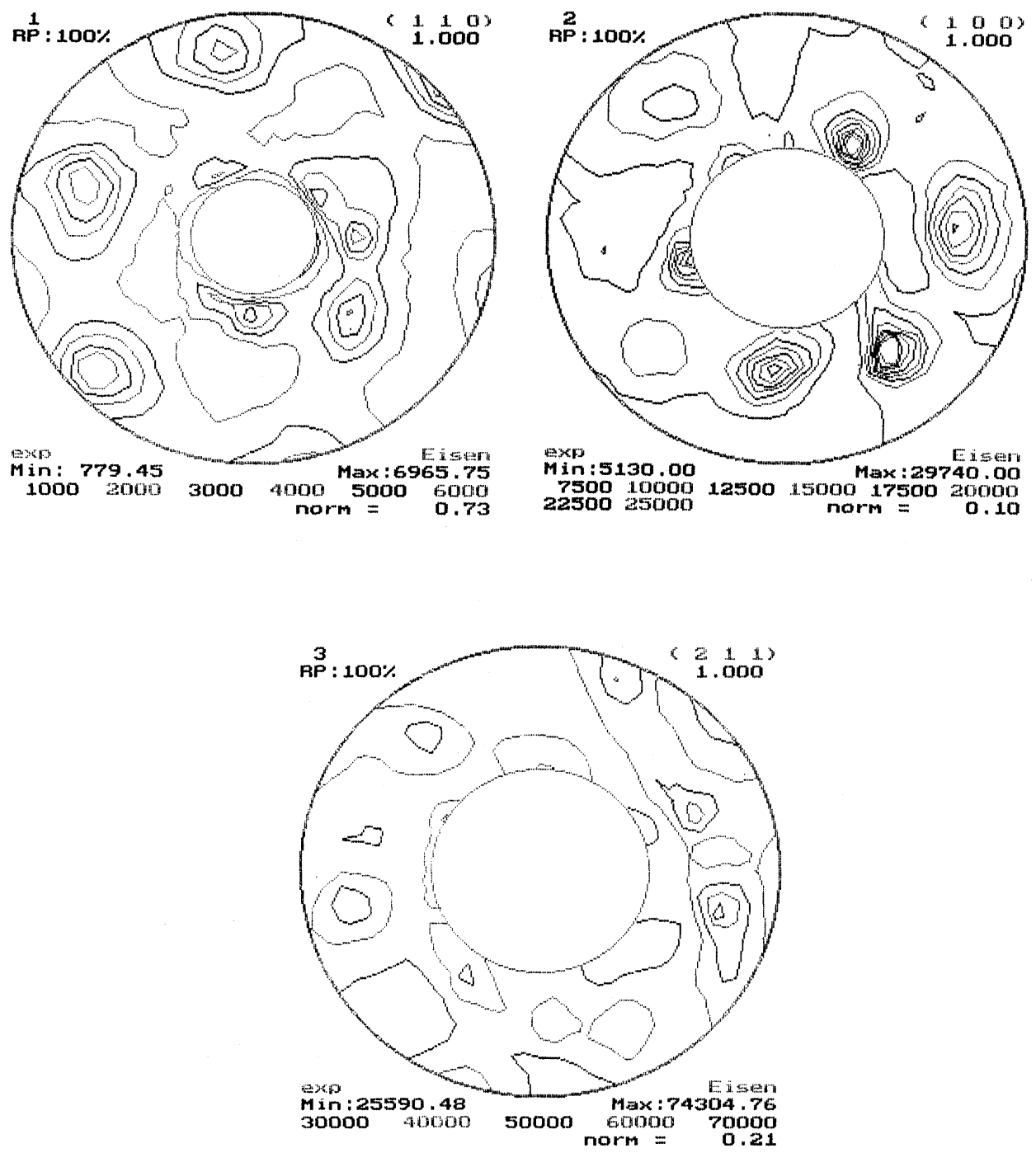

Figure 1. Pole figures (110), (200) and (211) of meteoric material (iron-nickel meteor). 
TEXTURE ANALYSIS

The calculation of the ODF was done with a computer routine using standard functions and a new minimal pole density set method ${ }^{1}$.

The material shows a unexpected sharp texture. The time of flight through the dense atmospheric layers was short (maximal 10 sec.) thus the meteor fragment could not melt. The texture must be either a primarily one or has arisen during the bounce. As a preliminary result we found three components: $\quad g_{1}(\alpha, \beta, \gamma)=g_{1}(6,72,59), \quad g_{2}(61,53,59), \quad$ and $g_{3}(53,50,63)$. Their intensities are 50\%, $17 \%$ and $32 \%$.

Texture investigation of cosmic bodies have following peculiarities :

- Cosmic bodies have a very irregular form and therefore it is impossible to fix a sample coordinate system $K_{A}$ unambiguously. All components have therefore a degree of freedom due to any rotation of the sample coordinate system.

- The origin of the texture is unknown. Only the primary texture arising from deformation processes in the universe are from interest and must be distinguished from secondary processes during the bounce.

Metallographic investigation are going on to find the direction of maximal deformation which could be associated with the shock direction during the bounce on the earth. 
Furthermore experiments on shock deformation are planned with the aim to identify and exclude textures arisen from shock deformation. Further investigations are going on.

\section{ACKNOWLEDGEMENT}

The authors want to thank the Bashkirian Department of the Academy of Science for the meteoric sample. Also they want to thank the Central Institute of Nuclear Research Rossendorf for providing neutron diffraction facilities

\section{REFERENCE}

1 K. Helming, Minimal Pole Figure Ranges For Quantitative Texture Analysis, this proceedings 